\title{
Optimal Path Planning using Informed Probabilistic Road Map Algorithm
}

DOI:10.36909/jer.ASSEEE.16105

\author{
Muhammad Aria \\ Departemen Teknik Elektro, Universitas Komputer Indonesia, Indonesia
}

Email: muhammad.aria@email.unikom.ac.id; Corresponding Author

\begin{abstract}
This study aims to propose a new path planning algorithm that can guarantee the optimal path solution. The method used is to hybridize the Probabilistic Road Map (PRM) algorithm with the Information Search Algorithm. This hybridization algorithm is called the Informed-PRM algorithm. There are two informed search methods used. The first method is the informed sampling through an ellipsoid subset whose eccentricity is dependent on the length of the shortest current solution that is successfully planned in that iteration. The second method is to use a local search algorithm. The basic PRM algorithm will be run in the first iteration. Since the second iteration, the generation of sample points in the PRM algorithm will be carried out based on information. The informed sampling method will be used to generate $50 \%$ of the sampling points. Meanwhile, the remaining number of sample points will be generated using a local search algorithm. Using several benchmark cases, we compared the performance of the Informed-PRM algorithm with the Rapidly Exploring Random Tree* (RRT*) and informed RRT* algorithm. The test results show that the Informed-PRM algorithm successfully constructs the nearly optimal path for all given cases. In producing the path, the time and path cost of the Informed-PRM algorithm is better than the RRT* and Informed RRT* algorithm. The Friedman test was then performed to check for the significant difference in performance between Informed-PRM with RRT* and Informed RRT*. Thus, the Informed-PRM algorithm can be implemented in various systems that require an optimal path planning algorithm, such as in the case of medical robotic surgery or autonomous vehicle systems.
\end{abstract}




\section{INTRODUCTION}

Path planning algorithms are widely used in various applications such as automation (Friedrich, C. et al., 2018), robot navigation (Tsai, C. C. et al., 2011), driverless cars (Rasekhipour, Y. et al., 2016), digital character animation (Qiu, Z., and Liu, C. 2012), robotic surgery (Sudhakara, P. et al., 2018), and protein folding (Ekenna, C. et al., 2015). The main task of the path planning algorithm is to find a collision-free path for the agent to move from one point to another in the state spaces (Goyal, J. K., and Nagla, K. S. 2014). Various path planning algorithms have different performance/qualities. Some parameters that can be used to measure the performance of a path planning algorithm are the planning time and the cost path/optimality (Elbanhawi, M., and Simic, M. 2014; Yang, Y. et al., 2019). Therefore, a path planning algorithm is expected to provide optimal path solutions in a short time (Mashayekhi, R. et al., 2020a).

Path planning algorithms can be divided into graph-based path planning algorithms and sampling-based path planning algorithms (Ma, L. et al., 2015). The graph-based path planning algorithm will discretize the map at the beginning of the path planning process. This method is resolution optimal and resolution complete (Gammell, J. D. et al., 2015). Examples of graphical path planning algorithms are Dijkstra (Candra, A. et al., 2020) and A* (Liu, X., and Gong, D. 2011) algorithms. The disadvantage of this graphbased path planning algorithm is the difficulty in scaling problems with large dimensions or sizes (Strub, M. P., and Gammell, J. D. 2020). The sampling-based path planning algorithm does not discretize the map at the beginning of the path planning process. This method takes a random sample from the state space then checks the visibility of the sample to accept or reject it (Mashayekhi, R. et al., 2020a). The samplingbased algorithm can be used well on problems with large dimensions or sizes (Elbanhawi, M., and Simic, M. 2014). This method is probabilistically complete (Karaman, S., and Frazzoli, E. 2011). There are two types of sampling methods in this sampling-based algorithm: the multi-query method and the single-query method. An example of a path planning algorithm with a multi-query method is the Probabilistic Road Map (PRM) (Kumar, N. et al., 2016). In the PRM algorithm, they first create a road map by taking a random sample in the state space, then connecting the various sample locations with visibility. An example of a single-query path planner is Rapidly-exploring Random Tree (RRT) (Wang, Y., and Huang, Y. 2019). The RRT algorithm builds a search tree rooted from the initial location and explores the state space by 
growing the tree toward a random sample. But the RRT algorithm cannot provide an optimal solution (Aria, M. 2019). Therefore, the RRT* algorithm implements the choose-parent and re-wiring operations to optimize the resulting path (Karaman, S., and Frazzoli, E. 2011). But the RRT* algorithm samples the entire state space, making this method inefficient. The Informed RRT* algorithm limits the sampling area through an ellipsoid subset of the state space to provide a near-optimal and faster solution than RRT* (Gammell, J. D. et al., 2014). Another disadvantage of the single-query method is that to provide a nearoptimal solution, the number of samples must be increased. Therefore, in this paper, a hybridization process will be carried out between the PRM algorithm and the informed search method. The informed search method used was taken from the RRT* informed algorithm.

This research aims to propose a new path planning algorithm that can provide optimal path solutions. The method used is a hybridization of the PRM algorithm with an Information Search Algorithm. We call this algorithm the Informed-PRM algorithm. There is two informed search method used. The first method is informed sampling via a subset of ellipsoids whose eccentricity depends on the shortest current solution successfully planned in this iteration. This method is based on the Informed RRT* algorithm. The second method is to use a local search algorithm. First of all, the PRM algorithm will be repeated for several iterations. Furthermore, the generation of sample points in the PRM algorithm will be carried out based on information. The informed sampling method will be used to generate $50 \%$ of the sampling points.

Meanwhile, the remaining number of sample points will be generated using a local search algorithm. Then we compare the performance of the Informed-PRM algorithm with the RRT* and informed RRT* algorithm. The performance is compared to computation time and path cost. The test results show that the Informed-PRM algorithm successfully constructs the nearly optimal path for all given cases. In producing the path, the time and path cost of the Informed-PRM algorithm is better than the RRT* and Informed RRT* algorithm. Thus, the Informed-PRM algorithm can be implemented in various systems that require an optimal path planning algorithm. 


\section{METHOD}

The proposed algorithms are shown in Figure 1 and Figure 2. In the first iteration, the basic PRM algorithm will be executed (line 15 in algorithm 2). Since the second iteration, the generation of sample points in the PRM algorithm will be carried out based on information (lines $1-13$ in algorithm 2). The informed sampling method will generate $50 \%$ of the sampling points (lines $2-10$ in algorithm 2). Meanwhile, the remaining sample points will be generated using a local search algorithm (line 12 in algorithm 2). The informed search method was taken from the RRT* informed algorithm (Gammell, J. D. et al., 2014). In addition, the local search method used was taken from Aria's research (2020a).

Limiting the sampling point generation area is expected to improve the quality of the resulting paths for the same number of $\mathrm{n}$ nodes. The limitation of the sampling point generation area is also expected to reduce the computation time of the algorithm in producing near-optimal paths. The local search algorithm is used to check the possibility of getting a better path around the current best path. This principle was also proposed by Aria (2020b). After the generation of sample points has been completed, the process of finding the best path from the roadmap provided will be carried out using the Djikstra algorithm (line 21 in algorithm 1)

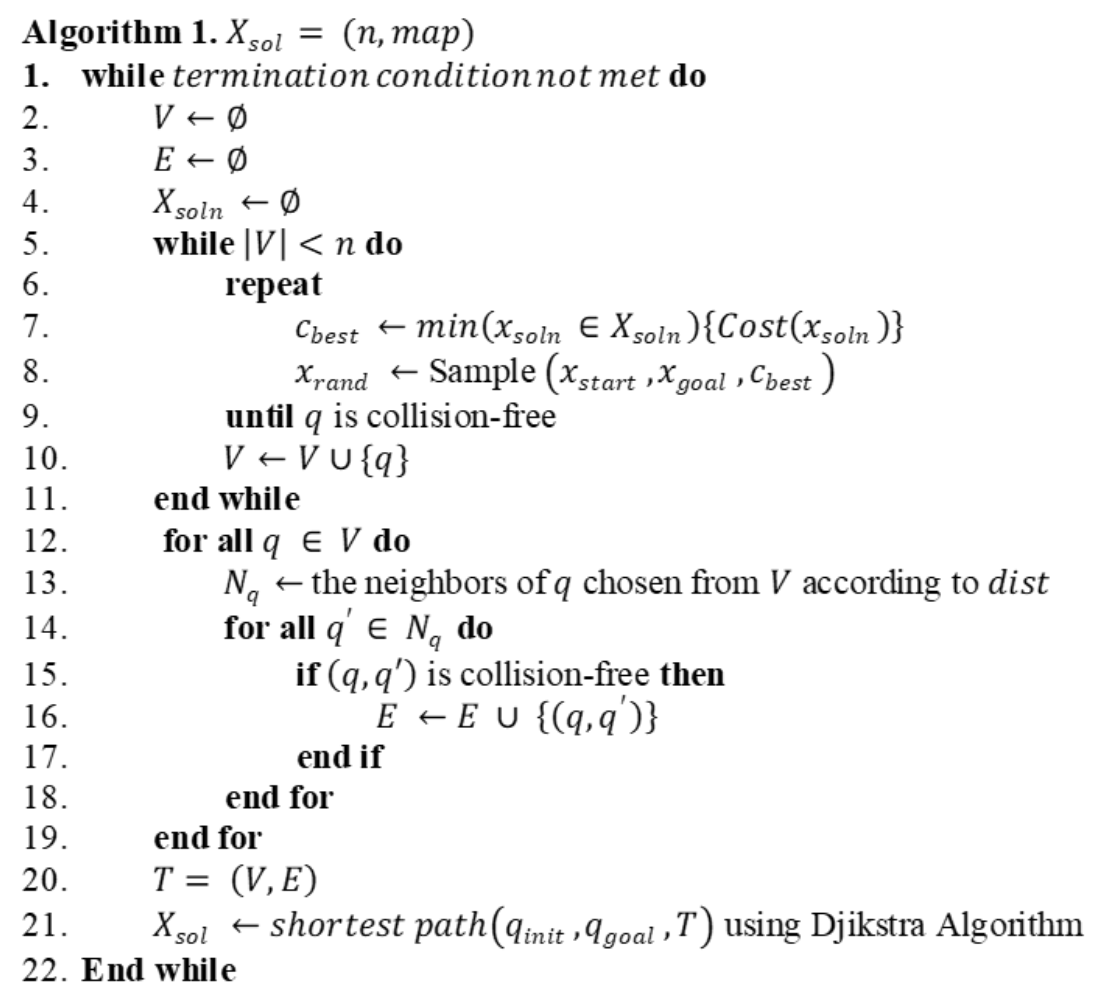


Figure 1 Informed-PRM algorithm

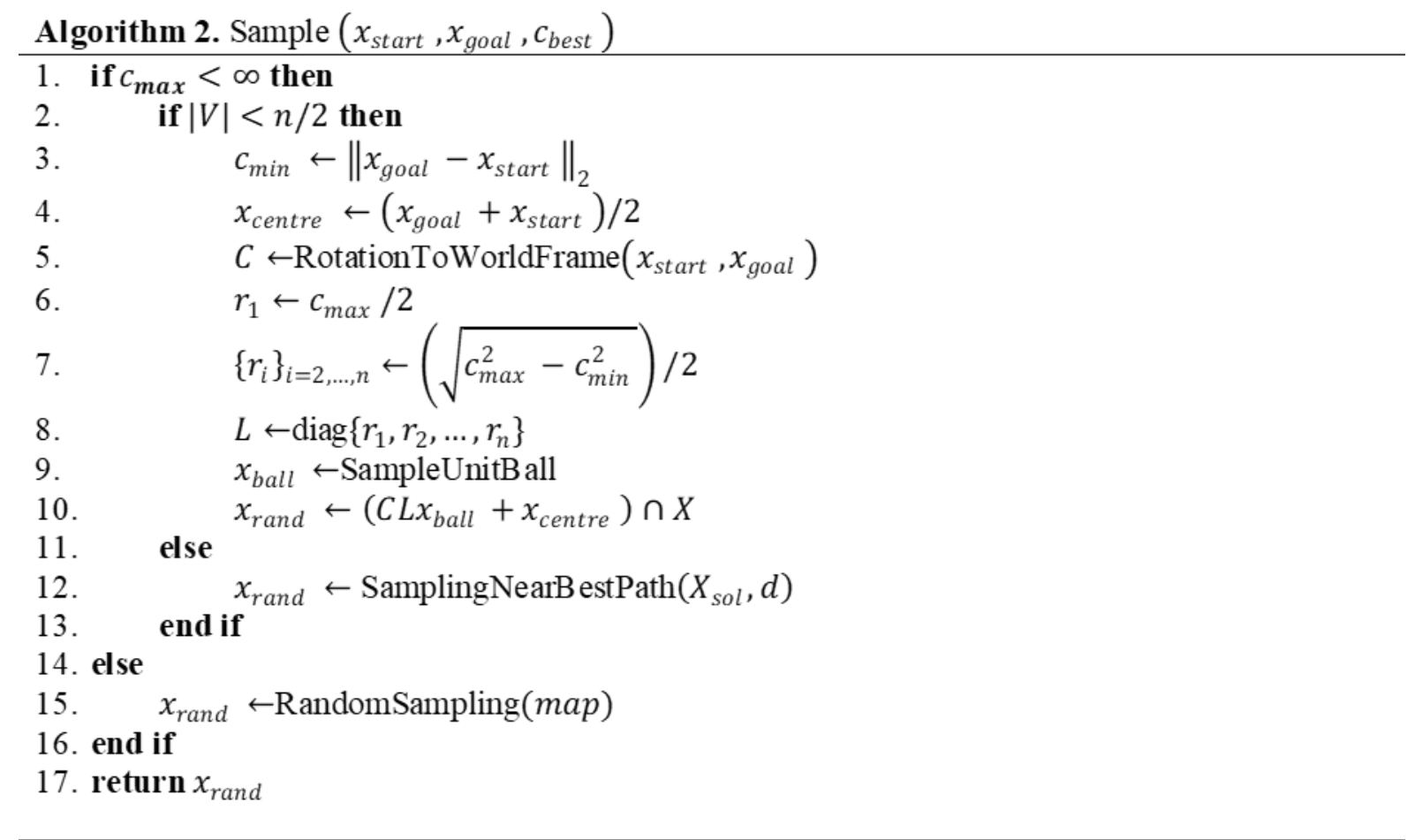

Figure 2 Propose an informed search method

\section{RESULTS AND DISCUSSION}

Four different experimental scenarios are presented in this section. These experiments were carried out to demonstrate the proposed Informed-PRM in solving the path planning problem. All experiments and analyses were carried out using the same PC (with a Core i5 $3.20 \mathrm{GHz}$ CPU and 4GB RAM) with Windows 10 64-bit Professional. LabVIEW 7 programming language is used to implement the proposed algorithm and other comparison algorithms.

\section{Verification for the Effectiveness of The Proposed Strategies}

In the first experiment, we will discuss the effectiveness of adding an informed search method to the PRM algorithm. The Informed-PRM algorithm (the proposed algorithm) is a hybridization algorithm of the PRM algorithm (Kumar, N. et al., 2016) and the Informed-RRT* algorithm (Gammell, J. D. et al., 2014). So we tested whether there was an increase in performance due to the addition of the informed search method to the PRM algorithm compared to the performance of the normal PRM algorithm and the Informed-RRT* algorithm. 
The experiment is carried out in a narrow environment, as shown in Figure 3. The Informed-PRM algorithm and the PRM algorithm run 100 times, each with 100 iterations (with an average time of 16.69 $\mathrm{ms}$ ). The Informed-RRT* algorithm runs 100 times, each for $16.69 \mathrm{~ms}$ (proportional to the Informed-PRM algorithm to perform 100 iterations). The statistical results of the performance comparison between Informed-PRM, PRM, and Informed-RRT* in the narrow environment case are shown in Table 1. An example of the path results generated by each algorithm is shown in Figure 3.

From Table 1, it can be seen that the Informed-PRM algorithm can consistently produce optimal paths ( standard deviation value $=0$ ). From Table 1, it can also be seen that the addition of the informed search method to the normal PRM algorithm has succeeded in improving the performance of the normal PRM algorithm. In Table 1, it is shown that the PRM algorithm cannot always produce an optimal path (standard deviation value $=1.54$ ), so after adding the informed search method (becoming the Informed-PRM algorithm), it can produce the optimal path. Table 1 shows that although the idea of the Informed-PRM algorithm is based on the Informed-RRT* algorithm. The performance of the Informed-PRM algorithm seems to be competitive with the Informed-RRT* algorithm (the mean and standard deviation of the Informed-PRM algorithm is less than the Informed-RRT* algorithm for the narrow environment). Performance comparisons between the informed-PRM algorithm and the informed-RRT * algorithm for various other cases will be presented in the third experiment.

Table 1 Performance Comparison between Informed-PRM, PRM and Informed-RRT* Using Narrow Environment

\begin{tabular}{|c|c|c|c|c|}
\hline Algorithms & Best & worst & mean & std \\
\hline $\begin{array}{c}\text { Informed-PRM } \\
\text { (100 iterations, with an } \\
\text { average time of } 16.69 \\
\text { ms) }\end{array}$ & $\mathbf{4 2 7 , 1 6}$ & $\mathbf{4 2 7 , 1 6}$ & $\mathbf{4 2 7 , 1 6}$ & $\mathbf{0}$ \\
\hline $\begin{array}{c}\text { PRM } \\
\text { (100 iterations) }\end{array}$ & 430,18 & 436,37 & 433,66 & 1,54 \\
\hline $\begin{array}{c}\text { Informed-RRT* } \\
\text { (Average running time } \\
\text { 16.69 ms) }\end{array}$ & 427,65 & 429,65 & 428,21 & 0,5 \\
\hline
\end{tabular}

The effectiveness of the Informed-PRM algorithm can also be seen from the path image shown in Figure 3. The best path should turn only at the corner of obstacles (Liu, Y. T. et al., 2020). The path generated by the Informed-PRM algorithm succeeds in only turning at the corner of obstacles, as shown in 
Figure 3 (a). However, the path generated by the PRM and Informed-RRT * algorithms, during the mean running time of $16.69 \mathrm{~ms}$, has not yet touched the corner of the obstacle, as shown in Figure 3 (b) and Figure 3 (c).

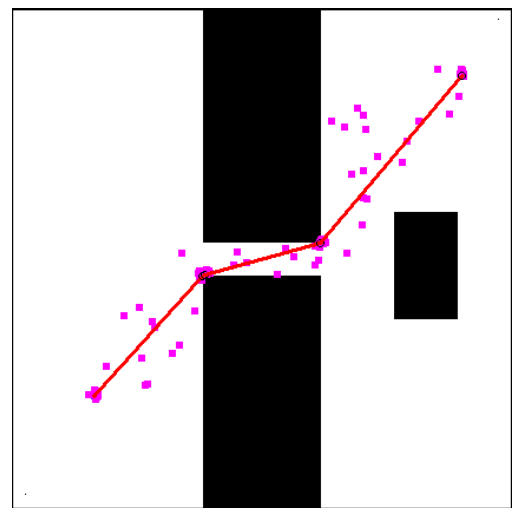

(a)

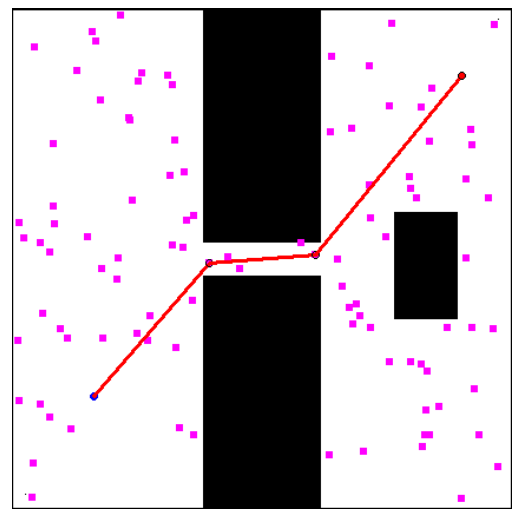

(b)

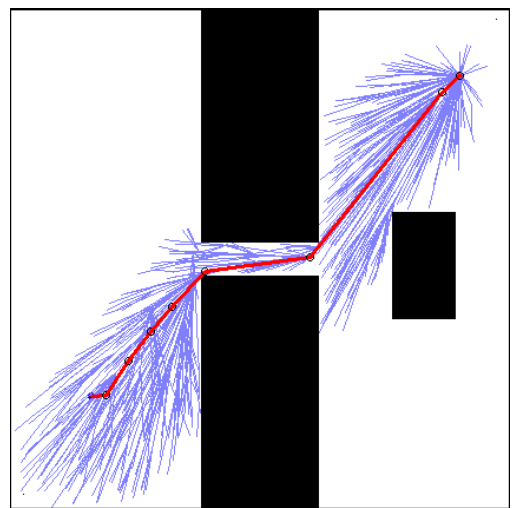

(c)

Figure 3 Path comparisons generated by each algorithm: (a) Informed-PRM algorithm after 100 iterations, (b) PRM algorithm after 100 iterations, and (c) Informed-RRT * algorithm after $16.69 \mathrm{~ms}$ (proportional to the meantime of Informed-PRM algorithm to perform 100 iterations).

Figure 3 also shows how this informed search method works. In the PRM algorithm (Figure 3b), the sampling points are evenly distributed over the entire search space. Whereas in the Informed-PRM algorithm (Figure 3a), the sampling points are limited to an ellipsoid subset whose eccentricity depends on the length of the best path solution. The idea of limiting point sampling is based on an informed-PRM algorithm that also limits its search tree to ellipsoid areas (Figure 3c).

\section{Effect of $\gamma$ on Informed-PRM Performance}

In previous experiments, the informed sampling method will generate $50 \%$ of the sampling points. Meanwhile, the remaining number of sample points will be generated using a local search algorithm. In this second experiment, we will observe whether there is a change in the performance of the informedPRM algorithm if the comparison of the number of samples generated by the informed sampling method and the local search method is changed. The comparison constant between the number of samples generated by the informed sampling method to the number of samples generated by the local search method is called $\gamma$.

Experiments were carried out for nine different $\gamma$ values. Each run 100 times with a limited number of iterations. Table 2 shows the values of Quartile 3, Median, and Quartile 1 of the path cost for each $\gamma$ value. When $\gamma$ is between 0.1 and 0.3 , the greatest path cost is obtained from the test results. Meanwhile, when 
the gamma value is more than 0.5 , the smallest path cost is obtained. It shows that the role of the sampling points method using the local search method is very important in improving the performance of the Informed-PRM algorithm.

Table 2 The Values of Quartile 3, Median, and Quartile 1 of The Path Cost for Each $\gamma$ Value

\begin{tabular}{|c|c|c|c|c|c|c|c|c|c|}
\hline \multirow{2}{*}{$\begin{array}{l}\text { Performance } \\
\text { measurement }\end{array}$} & \multicolumn{9}{|c|}{$\gamma$ value } \\
\hline & 0.1 & 0.2 & 0.3 & 0.4 & 0.5 & 0.6 & 0.7 & 0.8 & 0.9 \\
\hline Quartile 1 & 430.96 & 428.84 & 428.37 & 429.46 & 428.73 & 428.55 & 428.67 & 428.81 & 429.00 \\
\hline Median & 437.32 & 432.29 & 431.60 & 431.01 & 430.84 & 429.99 & 429.74 & 429.93 & 429.94 \\
\hline Quartile 3 & 441.49 & 433.04 & 433.52 & 431.69 & 432.65 & 431.08 & 430.67 & 431.07 & 430.18 \\
\hline
\end{tabular}

\section{Performance Comparison Between Informed-PRM and Other Algorithms}

To study the efficiency of the Informed-PRM algorithm (proposed algorithm) algorithm in the path planning problem, the third experiment is carried out to compare the performance of the Informed-PRM algorithm against the RRT* and Informed RRT* algorithms. The test case is taken from several references regarding path planning research. Map 1 is no obstacle environment. Map 2 is an obstacle environment. Map 1 and Map 2 are taken from Karaman's research paper when publishing the RRT* algorithm (2011). Map 3 and Map 4 is clutter environment, taken from Gammel's research paper when publishing the Informed-RRT* algorithm (2014). Map 5 is a tough passage environment. Map 6 is a square field environment. Map 5 and Map 6 are taken from Klem's research paper when publishing the RRT*-connect algorithm (2015). Map 7 is a cube with narrow passages. Map 8 is multiple narrow passages. Map 7 and Map 8 are taken from Mashayekhi's research paper when publishing the Informed RRT*- connect algorithm (2020b).

The performance criteria measured and compared are path costs. All algorithms are run 100 times, each with 10,000 iterations. The Informed-PRM algorithm is run 100 times, each with 100 iterations. The RRT* algorithm and the Informed-RRT* algorithm are executed 100 times, each with a time limit proportional to the Informed-PRM algorithm to perform 100 iterations. The statistical results of the performance comparison are shown in Table 3. 
Table 3 Each algorithm obtained statistical results on a different map. The best results for each map are highlighted in bold.

\begin{tabular}{|c|c|c|c|c|c|}
\hline Environment & Algorithms & Best & worst & mean & std \\
\hline \multirow{3}{*}{ Map 1} & Informed-PRM & 193,83 & 193,83 & 193,83 & $\mathbf{0}$ \\
\hline & RRT* & 194,38 & 193,85 & 194,04 & 0,17 \\
\hline & Informed-RRT* & 193,83 & 194,28 & 193,97 & 0,05 \\
\hline \multirow{3}{*}{ Map 2} & Informed-PRM & 344,93 & 344,93 & 344,93 & 0 \\
\hline & RRT* & 352,81 & 346,15 & 349,1 & 2,09 \\
\hline & Informed-RRT* & 349,28 & 345,71 & 347,02 & 1,25 \\
\hline \multirow{3}{*}{ Map 3} & Informed-PRM & 141,58 & 141,58 & 141,58 & $\mathbf{0}$ \\
\hline & RRT* & 146,12 & 142,01 & 144,17 & 1,48 \\
\hline & Informed-RRT* & 141,95 & 141,59 & 141,68 & 0,12 \\
\hline \multirow{3}{*}{ Map 4} & Informed-PRM & 142,05 & 142,13 & 142,09 & 0,04 \\
\hline & RRT* & 146,54 & 142,34 & 143,97 & 1,64 \\
\hline & Informed-RRT* & 142,01 & 141,71 & 141,81 & 0,11 \\
\hline \multirow{3}{*}{ Map 5} & Informed-PRM & 432,02 & 432,02 & 432,02 & $\mathbf{0}$ \\
\hline & RRT* $^{*}$ & 451,89 & 441,32 & 446,15 & 3,18 \\
\hline & Informed-RRT* & 450,55 & 441,68 & 445,69 & 3,08 \\
\hline \multirow{3}{*}{ Map 6} & Informed-PRM & 473,27 & 473,27 & 473,27 & $\mathbf{0}$ \\
\hline & RRT* & 480,77 & 478,36 & 479,37 & 0,75 \\
\hline & Informed-RRT* & 482,44 & 476,01 & 479,32 & 2,2 \\
\hline \multirow{3}{*}{ Map 7} & Informed-PRM & 324,73 & 324,73 & 324,73 & $\mathbf{0}$ \\
\hline & RRT* & 328,41 & 326,45 & 327,34 & 0,58 \\
\hline & Informed-RRT* & 324,91 & 326,06 & 325,42 & 0,31 \\
\hline \multirow{3}{*}{ Map 8} & Informed-PRM & 393,61 & 393,61 & 393,61 & $\mathbf{0}$ \\
\hline & RRT $^{*}$ & 410,07 & 402,62 & 406,66 & 0,58 \\
\hline & Informed-RRT* & 405,16 & 399,85 & 402,78 & 1,6 \\
\hline
\end{tabular}

It can be seen from Table 3 that the Informed-PRM algorithm can find the minimum path for almost all cases. For the case of map 4 (clutter environment), the Informed-RRT* algorithm can produce a shorter path than the informed-PRM algorithm. However, the standard deviation value of the informed-PRM algorithm in the case of map 4 is still better. In other cases, the RRT* algorithm and the informed-RRT* algorithm still need time to reach the minimum value. Generally speaking, the proposed Informed-PRM algorithm performs well overall in all of the environments.

Two special environments will be used to show a performance comparison of the computational speed of the informed-PRM algorithm. Two environments that are quite frequently used for testing path planning algorithms are the environment with obstacles and clutter environment. The environment with the obstacle test case is taken from Karaman's research paper when publishing the RRT* algorithm (2011). Gammel's research paper takes the cluttered environment test case when publishing the Informed-RRT* algorithm 
(2014). Figures 4 and 5 show a comparison of the minimum path costs and computational time of the Informed-PRM algorithm and RRT* algorithm for the environment with the obstacle test case. Figures 6 and 7 show a comparison of the minimum path costs and computational time of the Informed-PRM algorithm and Informed RRT* algorithm for clutter environment test case.

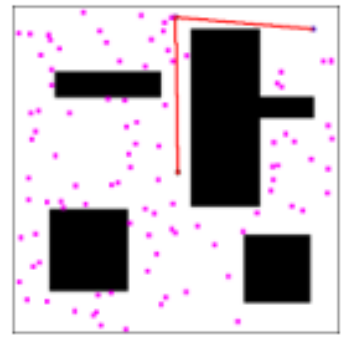

Time: $378 \mathrm{~ms}$ Cost : 378.65

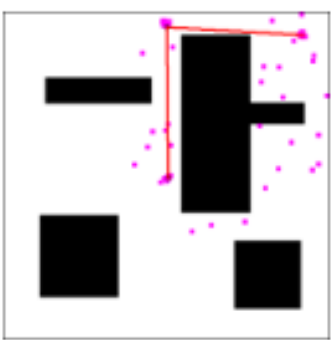

Time : $1067 \mathrm{~ms}$ Cost : 372.29

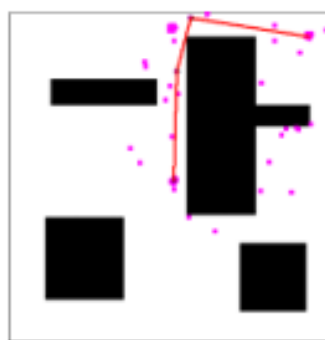

Time : $1755 \mathrm{~ms}$ Cost : 366.24

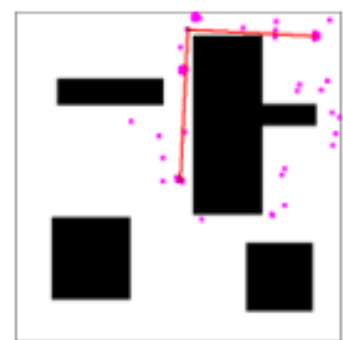

Time : $2301 \mathrm{~ms}$ Cost : 358.45

Figure 4 Minimum path costs and computation time of the Informed-PRM algorithm for the environment with obstacle

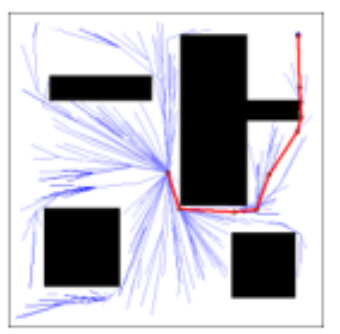

Time: $502 \mathrm{~ms}$ Cost : 405.55
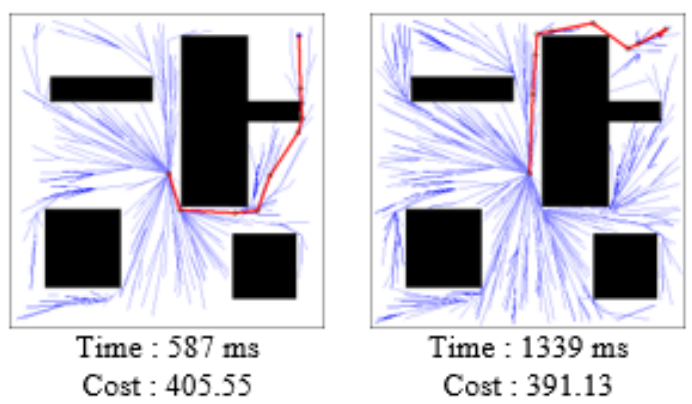

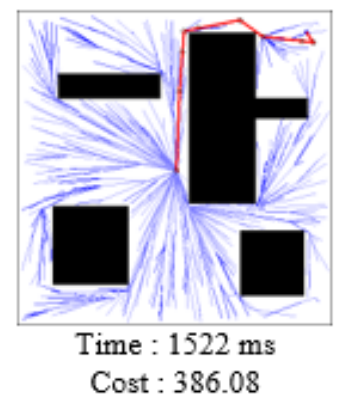

Cost : 386.08

Figure 5 Minimum path costs and computation time of the RRT* algorithm for the environment with obstacle
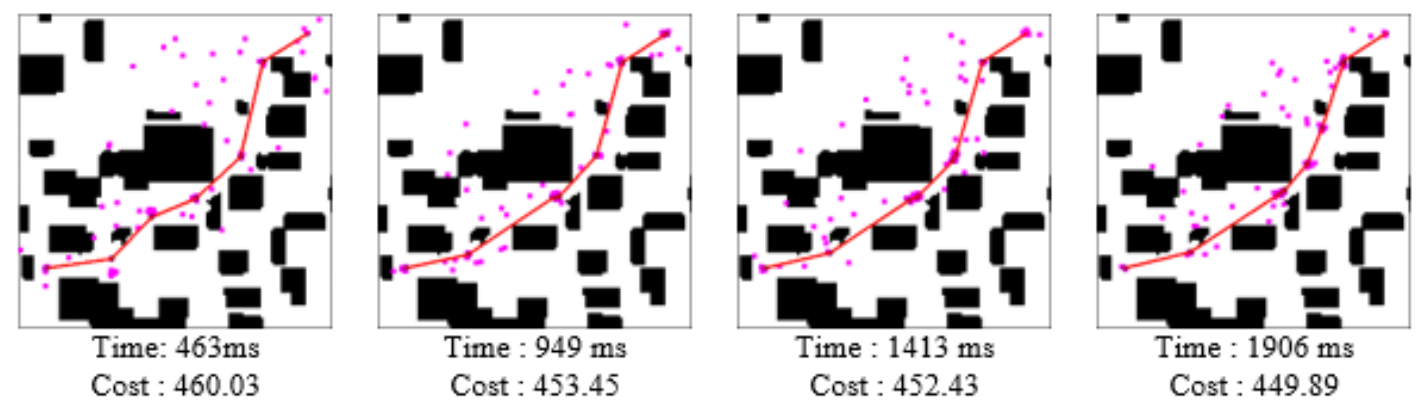

Figure 6 Minimum path costs and computation time of the Informed-PRM algorithm for clutter environment

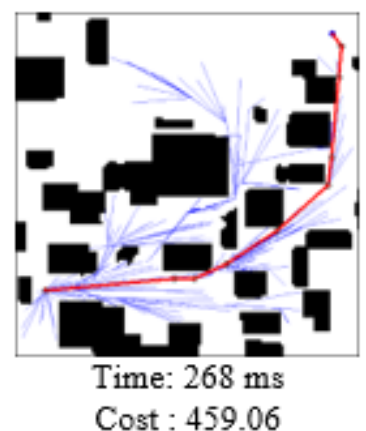

Cost : 459.06
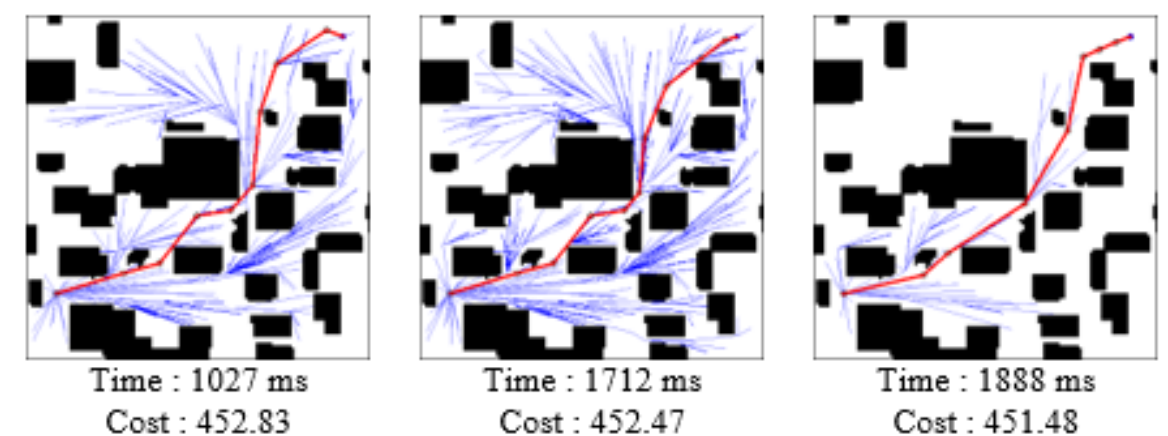
Figure 7 Minimum path costs and computation time of the informed RRT* algorithm for clutter environment

The results in Figure 5 are similar to those reported by Karaman, S., and Frazzoli, E. (2011), where the RRT* $^{*}$ algorithm will improve the quality of the resulting paths over time. The results in Figure 7 are also similar to those reported by Gammell, J. D. et al. (2014), where the Informed-RRT* algorithm will also improve the quality of the resulting paths over time.

From Figure 4 and Figure 5, it is perceived that the path cost and computation time required by the Informed-PRM algorithm is better than the path cost and computation time required by the RRT* algorithm for the case of an environment with the obstacle. And from Figure 6 and Figure 7, it can be seen that the path cost and computation time required by the Informed-PRM algorithm is better than the path cost and computation time required by the Informed-RRT* algorithm for the case of a cluttered environment. The Informed-PRM algorithm can have a good convergence speed because it limits the search area only in areas with a high chance of producing the optimal path based on the informed search method.

\section{Statistical Analysis of the Experimental Results}

Although the experimental results show that the informed-PRM algorithm is better than the RRT * and informed-RRT * algorithms, we cannot yet know whether there are significant differences between all methods due to the probabilistic characteristics of the algorithms. According to Derrac, J. et al. (2011) and Zhang, S. Et al. (2021), statistical tests should be carried out to improve the performance evaluation of different algorithms. Thus, we have performed two statistical tests with the results obtained in the previous subsection. The statistical software package SPSS was used to analyze the data in table 3 .

First, Friedman's non-parametric test was used to check whether there was a significant difference in performance among the three algorithms. The average ranking achieved by the statistical test for each compared algorithm can be seen in Table 4 (low rankings indicate better performance). The Friedman statistic obtained is 14,25 . Given that the confidence interval has been set at $95 \%$, the critical point is 11,07. Since $14,25>11,07$, it can be concluded that there is a statistically significant difference between 
the three algorithms based on the mean rank returned by the Friedman test. The informed-PRM algorithm can be considered as the method that has the lowest ranking.

Table 4 Average rankings obtained by Friedman's nonparametric test.

\begin{tabular}{|c|c|}
\hline Algorithm & Average Rangking \\
\hline Informed-PRM & 1,13 \\
\hline RRT $^{*}$ & 3 \\
\hline Informed-RRT & 1,88 \\
\hline
\end{tabular}

The results from the Friedman test only show whether there are significant differences but do not indicate which groups are particularly different from each other. Thus, to better evaluate the performance of the informed-PRM, we present a test in which the informed-PRM algorithm will be compared with the RRT $^{*}$ algorithm and the informed-RRT* algorithm using a multiple comparison procedure. Table 5 summarizes the results of these comparisons. From the results reported in Table 5, we can see that the significance value between the informed-PRM algorithm and the RRT* algorithm is 0.017 . This value is lower than 0.05 . So the informed-PRM algorithm is significantly better than the RRT* algorithm at the 95\% confidence level. Although the informed-PRM algorithm is not significantly better than the informedRRT* algorithm, the informed-PRM algorithm still performs better according to the mean values shown in Table 3.

Table 5 Test Summary Between Informed-PRM with RRT* and Informed-RRT*

\begin{tabular}{|c|c|}
\hline Informed-PRM vs & Sig. \\
\hline RRT $^{*}$ & 0,017 \\
\hline Informed-RRT $^{*}$ & 0,316 \\
\hline
\end{tabular}

\section{CONCLUSION}

The Informed-PRM algorithm succeeded in designing the nearly optimal path for each benchmark. The test results show that the computation time and path cost of the Informed-PRM algorithm is better than the RRT* and Informed RRT* algorithm for an environment with obstacles and a cluttered environment. The Informed-PRM algorithm can have a good convergence speed because it limits the search area only in areas with a high chance of producing the optimal path based on the informed search method. And based on the test results, the role of the sampling points method using the local search method is very important 
in improving the performance of the Informed-PRM algorithm. In addition, Frieman's non-parametric test is also applied to demonstrate the effectiveness of the proposed algorithm more scientifically. Thus, the Informed-PRM algorithm can be implemented in various systems that require an optimal path planning algorithm, such as in the case of medical robotic surgery or autonomous vehicle systems.

\section{ACKNOWLEDGMENT}

We would like to thank Universitas Komputer Indonesia for supporting this journal.

\section{REFERENCES}

Aria, M. 2019. New Sampling based planning algorithm for local path planning for autonomous vehicles. Journal of Engineering Science and Technology Special Issue on INCITEST 2019 66-76

Aria, M. 2020a. Path Planning Algorithm for Autonomous Vehicles Based on Hybridization of BFS and Path Smoothing Algorithm. Jurnal Telekontran. 8(1), 13-22

Aria, M. 2020b. Path Planning Algorithm using Informed Rapidly Explorign Random Tree*-Connect with Local Search. Journal of Engineering Science and Technology Special Issue on INCITEST 2020. 5057

Candra, A., Budiman, M. A., and Hartanto, K. 2020. Dijkstra's and A-Star in Finding the Shortest Path: a Tutorial. 2020 International Conference on Data Science, Artificial Intelligence, and Business Analytics (DATABIA). 28-32

Derrac, J., García, S., Molina, D., and Herrera, F. 2011. A practical tutorial on the use of nonparametric statistical tests as a methodology for comparing evolutionary and swarm intelligence algorithms. Swarm and Evolutionary Computation. 1(1): 3-18.

Ekenna, C., Thomas, S., and Amato, N. M. 2015. Adaptive local learning in sampling based motion planning for protein folding. IEEE International Conference on Bioinformatics and Biomedicine (BIBM), 61-68

Elbanhawi, M., and Simic, M. 2014. Sampling-based robot motion planning: A review. IEEE Access. 2: $56-77$ 
Friedrich, C., Csiszar, A., Lechler, A., and Verl, A. 2018. Efficient task and path planning for maintenance automation using a robot system. IEEE Transactions on Automation Science and Engineering. 15(3): 1205-1215

Gammell, J. D., Srinivasa, S. S., and Barfoot, T. D. 2014. Informed RRT*: Optimal sampling-based path planning focused via direct sampling of an admissible ellipsoidal heuristic. 2014 IEEE/RSJ International Conference on Intelligent Robots and Systems. 2997-3004

Gammell, J. D., Srinivasa, S. S., and Barfoot, T. D. 2015. Batch informed trees (BIT*): Sampling-based optimal planning via the heuristically guided search of implicit random geometric graphs. 2015 IEEE international conference on robotics and automation (ICRA). 3067-3074

Goyal, J. K., and Nagla, K. S. 2014. A new approach of path planning for mobile robots. 2014 International Conference on Advances in Computing, Communications and Informatics (ICACCI). $863-867$

Karaman, S., and Frazzoli, E. 2011. Sampling-based algorithms for optimal motion planning. The International Journal of Robotics Research. 30(7): 846-894

Kumar, N., Vámossy, Z., and Szabó-Resch, Z. M. 2016. Robot path pursuit using probabilistic roadmap 2016. IEEE 17th International Symposium on Computational Intelligence and Informatics (CINTI), 000139-000144

Liu, X., and Gong, D. 2011. A comparative study of A-star algorithms for search and rescue in perfect maze. 2011 International Conference on Electric Information and Control Engineering, 24-27

Liu, Y. T., Sun, R. Z., Zhang, T. Y., Zhang, X. N., Li, L., \& Shi, G. Q. 2020. Warehouse-Oriented Optimal Path Planning for Autonomous Mobile Fire-Fighting Robots. Security and Communication Networks, 2020: 6371814. https://doi.org/10.1155/2020/6371814

Ma, L., Xue, J., Kawabata, K., Zhu, J., Ma, C., and Zheng, N. 2015. Efficient sampling-based motion planning for on-road autonomous driving. IEEE Transactions on Intelligent Transportation Systems. 16(4): 1961-1976

Mashayekhi, R., Idris, M. Y. I., Anisi, M. H., and Ahmedy, I. 2020a. Hybrid RRT: A Semi-Dual-Tree RRT-Based Motion Planner. IEEE Access. 8: 18658-18668. 
Mashayekhi, R., Idris, M. Y. I., Anisi, M. H., Ahmedy, I., and Ali, I. 2020b. Informed RRT*-connect: An asymptotically optimal single-query path planning method. IEEE Access. 8: 19842-19852.

Qiu, Z., and Liu, C. 2012. The motion planning in the automatic generation of mobile phone 3D animation. Proceedings of the 10th World Congress on Intelligent Control and Automation. 725-731

Rasekhipour, Y., Khajepour, A., Chen, S. K., and Litkouhi, B. 2016. A potential field-based model predictive path-planning controller for autonomous road vehicles. IEEE Transactions on Intelligent Transportation Systems. 18(5): 1255-1267

Strub, M. P., and Gammell, J. D. 2020. Advanced BIT*(ABIT*): Sampling-Based Planning with Advanced Graph-Search Techniques. 2020 IEEE International Conference on Robotics and Automation (ICRA). 130-136

Sudhakara, P., Ganapathy, V., and Sundaran, K. 2018. Trajectory Planning Using Enhanced Probabilistic Roadmaps For Pliable Needle Robotic Surgery. 2018 International Conference on Recent Trends in Electrical, Control and Communication (RTECC). 61-64

Tsai, C. C., Huang, H. C., and Chan, C. K. 2011. Parallel elite genetic algorithm and its application to global path planning for autonomous robot navigation. IEEE Transactions on Industrial Electronics. 58(10):,4813-4821

Wang, Y., and Huang, Y. 2019. Mobile Robot Path Planning Algorithm Based on Rapidly-Exploring Random. Tree 2019 IEEE International Conferences on Ubiquitous Computing \& Communications (IUCC) and Data Science and Computational Intelligence (DSCI) and Smart Computing, Networking and Services (SmartCNS). 555-560

Yang, Y., Pan, J., and Wan, W. 2019. Survey of optimal motion planning. IET Cyber-systems and Robotics. 1(1): 13-19.

Zhang, S., Pu, J., \& Si, Y. 2021. An Adaptive Improved Ant Colony System Based on Population Information Entropy for Path Planning of Mobile Robot. IEEE Access. 9: 24933-24945. 